\title{
EGFR gets in the way of microRNA biogenesis
}

\author{
Cell Research (2013) 23:1157-1158. doi:10.1038/cr.2013.87; published online 9 July 2013
}

Recent studies suggest that microRNA (miRNA) processing is a key regulatory step in the miRNA biogenesis as well as its transcriptional control. In a paper recently published in Nature, Shen et al. revealed that epidermal growth factor receptor (EGFR) directly interacts with argonaute 2 (AGO2), a critical component of RNA-induced silencing complex (RISC), and inhibits the maturation of specific tumor suppressive miRNAs under hypoxic condition.

Two decades after the miRNA discovery, miRNA research has been growing rapidly towards diverse directions. One of the most important topics is the complexity of their biogenesis. The miRNA sequence is originally transcribed from host gene by RNA polymerase II ( $\mathrm{Pol}$ II), partially by RNA polymerase III (Pol III), as primary transcript (pri-miRNA). Pri-miRNAs are then processed by the ribonuclease III (RNase III) type endonuclease Drosha and its partner protein complex (Drosha microprocessor complex), which generates 70-nt stem loop transcripts known as precursor miRNAs (pre-miRNAs). After exported from the nucleus to the cytoplasm, Dicer, another RNase III type endonuclease, cleaves pre-miRNAs, generating 22-nt mature form of miRNAs [1] (Figure 1). Mature miRNAs exert its activity within RISC, which includes $\mathrm{AGO} 2$ protein, a crucial component for miRNA binding. miRNAs recognize its target mRNA in a sequence-dependent manner and suppress the target expression by destabilization of the transcripts and translational repression [1]. So far, several upstream regulators of miRNA processing have been identified. Tumor suppressor protein $\mathrm{p} 53$ directly interacts with RNA helicase p68, a component of the Drosha microprocessor complex, and facilitates the processing of pri-miRNAs to premiRNAs in response to DNA damage [2]. p68 is also shown to be targeted by SMAD, transforming growth factor $\beta$ (TGF $\beta)$ and bone morphogenetic protein (BMP) signaling transducer, and promotes the maturation of pri-miR-21 [3]. As other well-understood example, RNA-binding protein Lin 28 selectively blocks the processing of pri-let-7 miRNAs by inducing uridylation of let-7 precursor (pre-let-7) [4]. These studies clearly showed miRNA biogenesis is systematically controlled by upstream regulators.

A recent report by Shen et al. [5] elegantly revealed that EGFR, a wellcharacterized oncogene in human cancers, suppresses the maturation of specific miRNAs in response to hypoxic stress. EGFR is a cell surface protein, but is found to interact with $\mathrm{AGO} 2$ in cytoplasm through enhanced intracellular vesicle formation called internalization under hypoxic condition.

The association between EGFR and AGO2 leads to the elevated AGO2Y393 phosphorylation, which in turn reduces the binding of Dicer to AGO2. As Dicer cleaves precursor miRNAs and generates mature miRNAs, the reduced binding results in an inhibition of miRNA biogenesis (Figure 1). Interestingly, the majority of the miRNAs inhibited by EGFR turned out to have tumor suppressor characteristics, suggesting that EGFR exert its oncogenic property through inhibition of miRNA processing. Importantly, higher expres- sion of phosphorylated-Y393-AGO2 was significantly correlated with poorer overall survival in human breast cancer samples. They also demonstrated that "a long loop structure" in the terminal loop of the pre-miRNA is crucial for the recognition and subsequent processing by Dicer in coordination with $\mathrm{AGO} 2$ and EGFR [5].

The study by Shen et al. provides two important suggestions. First, internalized EGFR could function as a crucial regulator of cellular function through miRNA regulation in response to hypoxic stress. Second, hypoxia directly influence the specific miRNA expression through modification of miRNA processing, instead of transcriptional control. Internalization of EGFR was shown to be a crucial event in the direct binding between EGFR and AGO2. Gibbings et al. [6] have previously reported in 2009 that $\mathrm{AGO} 2$ and other component of RISC, GW182, are often colocalized with multivesicular bodies (MVBs, intracellular vesicles originated by lipid bilayer), and demonstrated that RISC formation and/or turnover appears to occur on MVBs. This is supported by the result that blocking of MVB formation suppressed RISC activity and reduced the miRNA target inhibition [6]. The study by Shen et al. provides further insights into the interaction between cell surface protein and component of RISC. A part of MVBs can also fuse with the plasma membrane and finally release the inner vesicle to extracellular space as exosome [6]. Exosome is known to be enriched with miRNAs, and recent studies suggest the possibility that these miRNAs are transferred into recipient cells, where they function [7]. Consider- 


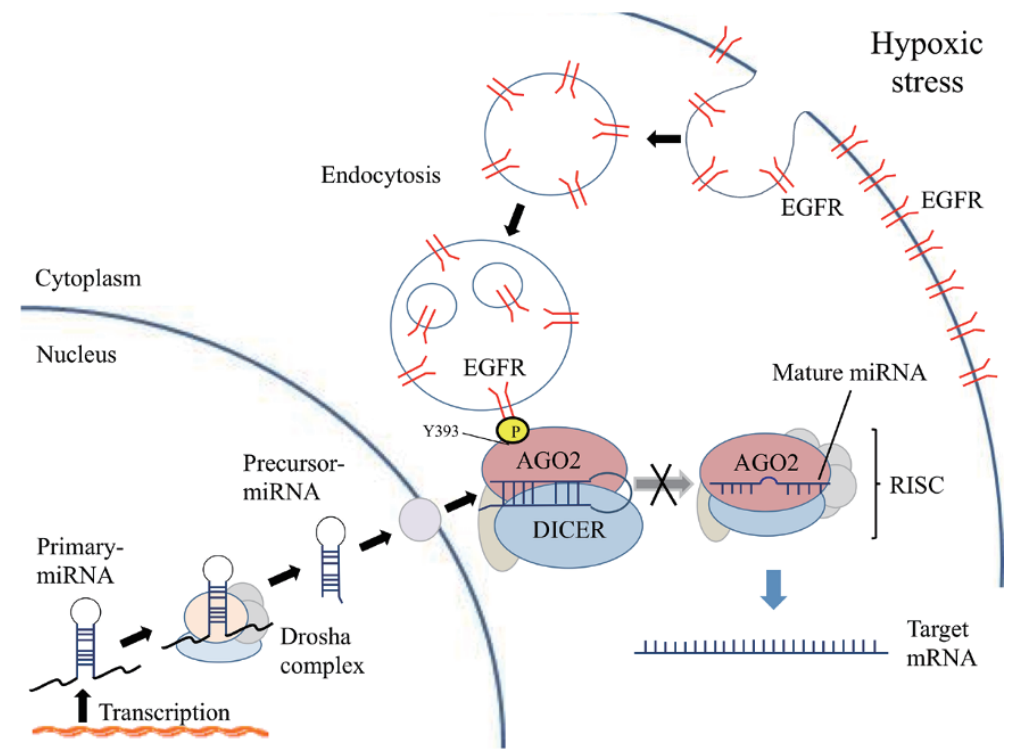

Figure $1 \mathrm{EGFR}$ inhibits the processing of precursor miRNA through phosphorylation of AGO2-Y393. miRNAs are transcribed from its host gene as primary miRNAs, and processed to 70-nt-long precursor miRNAs by endonuclease Drosha microprocessor complex. After active transportation to cytoplasm, Dicer, another RNase III type endonuclease, cleaves precursor miRNAs into short doublestranded RNA fragments in coordination with AGO2. Hypoxic stress induces the endocytic trafficking of EGFR into cytoplasm, where EGFR interacts with AGO2. EGFR reduces the binding of Dicer to AGO2 by phosphorylating AGO2-Y393, which results in an attenuated processing of precursor miRNAs to mature miRNAs.

ing the results of this study, the association between EGFR internalization and miRNA secretion into exosome could be a next study in the future.

Clear demonstration of the association between hypoxia and miRNA processing is intriguing. The alterations of miRNA expression under hypoxia have been mainly explained by transcriptional regulation, including DNA methylation of miRNA promoters. Hypoxia inducible factors (HIF) are known to play a central role, and some miRNAs are directly transcribed or indirectly activated by HIF [8]. However, in the study by Shen et al., regulation of miRNA expression is achieved by the processing control, instead of transcriptional activation. Stabilization of HIF $1 \alpha /$ HIF $2 \alpha$ was only required to trigger the interaction of EGFR and AGO2 [5]. Regarding AGO2 modification, another recent study demonstrated that hypoxia induces hydroxylation of AGO2, which in turn increases the endonuclease activity of $\mathrm{AGO} 2$ and promotes miRNA maturation [9]. AGO2 could potentially undergo a variety of post-transcriptional modifications (PTM) $[9,10]$, therefore future studies might further reveal the association between PTM of AGO2 and the efficiency of miRNA processing.

In conclusion, the study by Shen et al. sheds light on the novel intracellular function of EGFR in hypoxic condition. Regulation of miRNA processing by a well-characterized oncogene indicates that miRNA biogenesis is systematically regulated in response to cellular stress.

Naohiro Nishida ${ }^{1,2}$, Koshi Mimori ${ }^{3}$, Masaki Mori², George A Calin ${ }^{1}$

${ }^{1}$ Department of Experimental Therapeutics and The Center for RNA Interference and NonCoding RNAs, The University of Texas MD Anderson Cancer Center, 1515 Holcombe Boulevard, Houston, TX 77030, USA; ${ }^{2}$ Department of Gastroenterological Surgery, Osaka University Graduate School of Medicine, 2-2 Yamada-oka, Suita City, Osaka, Japan; ${ }^{3}$ Department of Surgery, Kyushu University Beppu Hospital, 4546 Tsurumibaru, Beppu, Oita, Japan Correspondence: George A Calin Tel: +1-713-792-5461; Fax: +1-713-745-4528 E-mail: gcalin@mdanderson.org

\section{References}

1 Inui M, Martello G, Piccolo S. Nat Rev Mol Cell Biol 2010; 11:252-263.

2 Suzuki HI, Yamagata K, Sugimoto K, et al. Nature 2009; 460:529-533.

3 Davis B, Hilyard A, Lagna G, et al. Nature 2008; 454:56-61.

4 Viswanathan SR, Daley GQ, Gregory RI. Science 2008; 320:97-100.

5 Shen J, Xia W, Khotskaya YB, et al. Nature 2013; 497:383-387.

6 Gibbings DJ, Ciaudo C, Erhardt M, et al. Nat Cell Biol 2009; 11:1143-1149.

7 Valadi H, Ekström K, Bossios A, et al. Nat Cell Biol 2007; 9:654-659.

8 Kulshreshtha R, Ferracin M, Wojcik SE, et al. Mol Cell Biol 2007; 27:1859-1867.

9 Wu C, So J, Davis-Dusenbery BN, et al. Mol Cell Biol 2011; 31:4760-4774.

10 Qi HH, Ongusaha PP, Myllyharju J, et al. Nature 2008; 455:421-424. 